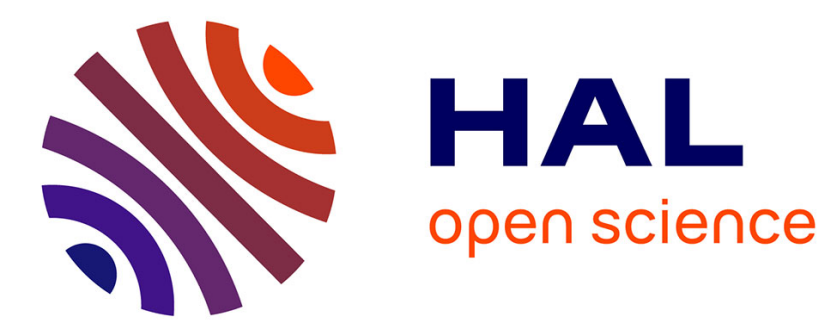

\title{
L'instrumentalisation par la preuve du corps du réfugié
}

Elise Pestre

\section{To cite this version:}

Elise Pestre. L'instrumentalisation par la preuve du corps du réfugié. Recherches en psychanalyse, 2012, Corps, identité et traumatisme / Body, Identity and Trauma, 2 (14), pp.147-154. 10.3917/rep.014.0147. hal-01507706

\section{HAL Id: hal-01507706 https://hal.science/hal-01507706}

Submitted on 13 Apr 2017

HAL is a multi-disciplinary open access archive for the deposit and dissemination of scientific research documents, whether they are published or not. The documents may come from teaching and research institutions in France or abroad, or from public or private research centers.
L'archive ouverte pluridisciplinaire HAL, est destinée au dépôt et à la diffusion de documents scientifiques de niveau recherche, publiés ou non, émanant des établissements d'enseignement et de recherche français ou étrangers, des laboratoires publics ou privés. 


\title{
L'INSTRUMENTALISATION PAR LA PREUVE DU CORPS DU RÉFUGIÉ
}

\author{
Élise Pestre
}

Association Recherches en psychanalyse | Recherches en psychanalyse

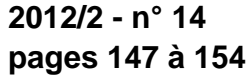

ISSN 1767-5448

Article disponible en ligne à l'adresse:

http://www.cairn.info/revue-recherches-en-psychanalyse-2012-2-page-147.htm

Pour citer cet article :

Pestre Élise, « L'instrumentalisation par la preuve du corps du réfugié »,

Recherches en psychanalyse, 2012/2 n 14, p. 147-154. DOI : 10.3917/rep.014.0147

Distribution électronique Cairn.info pour Association Recherches en psychanalyse.

(c) Association Recherches en psychanalyse. Tous droits réservés pour tous pays.

La reproduction ou représentation de cet article, notamment par photocopie, n'est autorisée que dans les limites des conditions générales d'utilisation du site ou, le cas échéant, des conditions générales de la licence souscrite par votre établissement. Toute autre reproduction ou représentation, en tout ou partie, sous quelque forme et de quelque manière que ce soit, est interdite sauf accord préalable et écrit de l'éditeur, en dehors des cas prévus par la législation en vigueur en France. II est précisé que son stockage dans une base de données est également interdit. 


\section{Recherches en Psychanalyse - Research in Psychoanalysis}

$14 \mid 2012$ - Corps, identité et traumatisme

Body, Identity and Trauma

\section{L'instrumentalisation par la preuve du corps du réfugié} Instrumentalizing the Refugee's Body Through Evidence

[En ligne] 28 décembre 2012

Élise Pestre

\section{Résumé :}

Le sujet qui demande l'asile au sein d'un nouvel État doit témoigner des craintes de persécutions qu'il encoure dans son pays. Une fois réalisé l'examen minutieux de son récit, les preuves corporelles de ses allégations seront appréciées par les administrations.

Les autorités porteront une attention toute particulière aux corps des requérants, dans l'objectif de valider - ou pas - leur témoignage, comme si les corps avaient le pouvoir de conserver à jamais les empreintes visibles des blessures passées. Cette quête souveraine de corps meurtris érige le soma en témoin incontournable du parcours d'asile du réfugié.

\section{Abstract :}

The subject who requests asylum in a new state must testify to his or her fears about the persecution he or she incurs in his or her country. Once a detailed examination of the account has been carried out, any corporeal evidence of his or her allegations will be scrutinized by the administrations.

The authorities will pay particular attention to the bodies of the asylum seekers, with the objective of confirming - or not - their account, as though the bodies had the power to conserve the visible imprints of past wounds forever. This sovereign quest for battered bodies sets up the soma as an indispensible witness to the journey towards asylum taken by the refugee.

Mots-clefs : réfugié, corps, trauma, biopolitique, instrumentalisation, globalisation, santé Keywords : refugee, body, trauma, biopolitics, instrumentalization, globalization, health

\section{Plan :}

État des lieux d'un problème d'actualité

La mise à l'épreuve juridique du corps du sujet

La hernie d'escale

Jouissance et maladie circulaire

Le corps dévoilé 
Lorsqu'un requérant demande l'asile sur le territoire français, les administrations concernées ${ }^{1}$ étudient minutieusement son récit afin d'en déterminer la véracité. Puis, elles évaluent les preuves relatives à ses allégations. Dans ce cadre, le corps du demandeur va faire l'objet $d$ 'une expertise médicale : la concordance entre ses propos et les traces de persécutions, quand elles sont mentionnées, seront dûment vérifiées par les autorités compétentes. ${ }^{2}$

Au cours de ce parcours, le corps du sujet va se configurer comme étant le témoin privilégié de ses persécutions et représenter la garantie majeure de sa vérité testimoniale. Nous envisagerons ici la façon dont les exigences politiques, et en particulier le régime d'instrumentalisation par la preuve, s'imbriquent à la problématique traumatique du sujet. La manière dont le corps pulsionnel du réfugié est " attrapé » par ce discours et dont il répond parfois symptomatiquement à ses attentes sera tout particulièrement exploré.

\section{État des lieux d'un problème d'actualité}

Depuis plusieurs décennies, en particulier avec l'avènement des années 1990 qui marquent l'expansion et l'assise de la globalisation, des lois restrictives sur les titres de séjour des étrangers se sont déployées en France, et audelà dans le monde. Le droit d'asile n'a pas été épargné par ces mesures nationales et internationales: aujourd'hui, lorsqu'un sujet s'exile en France, il s'avère difficile pour lui d'accéder au statut de réfugié. Environ $80 \%$ des demandeurs sont rejetés dans leur demande. ${ }^{3}$

L'argument politique avancé par l'État est sousjacent à sa volonté sécuritaire : "protéger » la Nation, l'autochtone, du xenos, l'étranger, dans l'objectif de préserver l'économie du pays. Appréhendés comme voulant "détourner la procédure $\|^{4}$ de l'asile, parias pauvres et dérangeants, ces supposés envahisseurs viennent néanmoins, selon les lois du capitalisme, servir les intérêts économiques du pays en participant directement à l'augmentation de la quantité des biens de la Nation, et de ses habitants.
Les administrations de l'État, à l'occasion du dépôt de la demande d'asile, sont en quête de I'authenticité du témoignage du requérant. Une sélection entre le "vrai » réfugié - qui serait plus idéalement le réfugié politique ${ }^{5}$, et le "faux", à savoir le migrant économique, s'engage alors. Pour autant, ces catégories se chevauchent parfois de manière complexe et subtile, la dimension économique de la migration recouvrant partiellement la dimension politique des persécutions. En effet, les catastrophes économiques d'un pays masquent fréquemment des conflits politiques et sociaux sous-jacents, les pays "en voie de développement " étant généralement ceux-là même où s'exercent des violences d'État. Pourtant, depuis l'avènement de la crise du droit d'asile, la dimension politique des persécutions est fréquemment évincée par les institutions au profit de l'économique, seule capable d'asseoir l'argument inégalable et absolu qui fonde le rejet de la majorité des demandes d'asile.

Avant de poursuivre plus en avant ces développements, et afin d'appréhender au mieux l'instrumentalisation par la preuve du corps du réfugié, il convient de mentionner quelques éléments récents notables de la littérature internationale. Si les recherches sur la santé des réfugiés mettent en évidence l'existence de nombreuses études épidémiologiques, l'état de santé de ces populations reste cependant peu systématisé, comme le remarque Gushulak B.-D ${ }^{6}$, . Au niveau du psychotraumatisme des réfugiés, la littérature se réfère dans la majorité des cas à la notion de PTSD (Post traumatic stress disorder) ${ }^{8}$ et propose des modalités de mesures relatives au déclenchement de ce syndrome ainsi qu'au repérage des signes dépressifs chez les migrants ayant subi des tortures. On remarque que, dans l'ensemble, et depuis les cinq dernières années, ces travaux quantitatifs se sont donc essentiellement penchés vers l'exploration des signes pathologiques et la recherche de techniques thérapeutiques efficientes auprès de ces populations. $^{9}$

Les avancées les plus fécondes se situent, nous semble-t-il, du côté de l'anthropologie médicale 
et politique, notamment avec les travaux de D. Fassin et R. Rechtman. ${ }^{10}$ Les études qui suscitent aussi de l'intérêt sont à relever dans les champs des gender studies ${ }^{11}$ évoqués, ou encore des global ou minority studies. ${ }^{12}$ Enfin, on mentionnera les importants travaux en psychiatrie transculturelle conduits au Canada par le psychiatre L.-J. Kirmayer. ${ }^{13}$ Une approche transdisciplinaire dans laquelle une psychodynamique du réfugié articule la psychopathologie psychanalytique à la médecine, et l'anthropologie à la philosophie politique est aussi à mentionner. ${ }^{14},{ }^{15}$ Cette contribution se propose de poursuivre précisément l'exploration de cette perspective clinique.

La mise à l'épreuve juridique du corps du sujet

Dans le cadre d'une étude qualititative menée auprès de divers professionnels qui travaillent avec de populations réfugiées (psychologues, médecins, travailleurs sociaux mais aussi des juristes, etc. $)^{16}$, l'analyse des entretiens de recherche montre que le demandeur d'asile qui présente des traces manifestes de persécutions, celui qui a été torturé, a finalement plus de chance d'être reconnu réfugié que celui qui ne l'a pas été. Cette considération amène à postuler que l'État attend par conséquent la production d'un état traumatique visible où la transparence des corps donnerait à voir un passé qui laisse des empreintes immuables, conservées à jamais, alors même que les marques liées aux tortures sont vouées à disparaître.

Dans ce contexte d'instrumentalisation des corps, de quelle façon la vie pulsionnelle du sujet de l'inconscient va-t-elle se relier à son parcours juridique et institutionnel ? Qu'est-ce que le discours politique engendre au niveau du corps du demandeur d'asile?

II nous semble important de souligner que, dans certaines configurations psychiques, traumatiques en particulier, le sujet en quête de refuge va se sentir parfois acculé, comme encouragé à montrer ses blessures psychiques et corporelles, pour démontrer à l'État les conséquences de ses persécutions, voire le ravage psychique qu'il traverse. Il va donc, à son insu le plus généralement, répondre à cette demande de preuves corporelles que lui adresse l'Autre. Cette sorte de prescription souveraine à être marqué est susceptible de retentir fortement en lui et se manifester par le déploiement d'une symptomatologie somatique massive qu'il mettra singulièrement en avant.

Aussi nous semble-t-il essentiel de souligner ici combien le corps biologique du réfugié apparaît capté, voir pénétré par la souveraineté politique et devient un objet de pouvoir. ${ }^{17}$ Cette conception avancée par Agamben s'articule à celle de la "disciplinarisation des corps ${ }^{18}$ dont parlait Foucault à l'occasion de ses développements sur le biopolitique. Cette stratégie de pouvoir étatique spécifique donne à voir l'extraordinaire pouvoir qu'exerce l'État sur les corps, et une forme d'assujettissement qu'il produit sur la vie des hommes.

Dans ce cadre, rappelons la "poussée » continue, au cours de cette dernière décennie, des demandes de certificats médicaux dans les centres de soins qui accueillent des réfugiés. La Direction du Comede ${ }^{19}$ a dû prendre des mesures pour que la pratique des médecins ne soit pas réduite à une répertoriation des symptômes de leurs patients, expertise issue de la demande de l'État. Devant l'inflation de ces demandes d'attestations diverses, un quota de cinq certificats maximum par jour a été instauré par la Direction. Aujourd'hui, expertiser, établir comme "compatibles" les déclarations d'un requérant à partir de l'observation de son corps est une activité devenue centrale de ces institutions de soins destinées à l'accueil des populations réfugiées. ${ }^{20}$

On pourrait penser que le patient en demande d'asile est donc convaincu - à juste titre ! - que le certificat médical délivré par son médecin attestera et appuiera auprès de l'État ses propos concernant les persécutions subies, qui suscitent tant d'incrédulité auprès des institutions. Car c'est bien en prouvant la teneur de ces atteintes physiques qu'il bénéficiera de chances optimales pour accéder à la qualité convoitée. 
On se rappelle à ce propos des mots d'un avocat qui allait plaider au Tribunal pour un demandeur d'asile, patient du Comede, et qui s'était dit " rassuré par le bon certificat de torture " qu'un médecin avait délivré à son "client». Cette formulation peu métaphorique laisse entrevoir les dérives éthiques relatives à cette nécessité implicite du requérant à être marqué. L'émergence d'un corps discipliné ${ }^{21}$ par l'institution, devenu obéissant et avili, amène le sujet à porter en offrande sa chair au souverain.

\section{La hernie d'escale}

Lorsque Lydie, une patiente demandeuse d'asile m'annonce au cours d'une séance le rejet de sa requête, elle fait spontanément le lien avec les propos de son avocat qui lui aurait demandé : "d'aller chercher d'autres preuves physiques" afin de mieux répondre aux volontés du Tribunal évoqué.

Le corps réel de Lydie est bel et bien atteint par la névrose: elle souffre de manière continue d'angoisses, de cauchemars, mais elle semble aussi affectée jusque dans ses organes par son exil et sa quête de refuge, vaine. Elle évoque des douleurs diverses: migraines, maux de dos et présente une pleurésie.

Lydie se plaint beaucoup de ce corps-qui-a-mal, sans répit, et à l'occasion d'un entretien, elle exprimera sa volonté de consulter un rhumatologue pour ses douleurs dorsales lancinantes. Si elle est francophone et s'exprime couramment en français, elle laissera néanmoins échapper, ce jour là, un formidable lapsus en voulant évoquer la douleur qui lui fait si mal au dos. Elle dira: "J'ai une hernie d'escale ".

Alors qu'elle est déboutée de l'asile, c'est désormais son corps qui devient LA possibilité, l'unique pour elle, de rester en France puisqu'elle devra pour obtenir des " papiers " demander un «droit au séjour pour raison médicale ${ }^{22} \mathrm{Et}$ pour l'obtenir elle n'aura d'autre choix que d'être malade et de continuer à l'être.

Cette patiente ne fait qu'asseoir auprès des autres, et en particulier des institutions souveraines, les symptômes corporels qui lui sont demandés. Cette situation clinique rappelle de près l'hypocondriaque qui "retire ses intérêts comme sa libido des objets du monde extérieur et concentre l'un et l'autre sur l'organe qui l'occupe $"{ }^{23}$

Cette demande étatique impérative "sert " sa névrose et se fixe sur certaines parties de son corps. Le médical entre ainsi dans l'économie pulsionnelle de sa maladie et réaménage sa trajectoire traumatique en direction de l'ordre juridique.

Les organes malades engendrent, malgré la douleur qu'ils produisent, un bénéfice direct pour le sujet. En se déployant sur la scène " externe ${ }^{24}$, ils offrent la possibilité réelle de rester sur le territoire français, et deviennent ainsi un gain " matériel, concret ", déterminant pour la survie. Cette forme de bénéfice se différencie de laformation de symptôme, interne et nécessaire, propre au fonctionnement névrotique du sujet; cet aspect n'en demeure pas moins central, mais vient se placer, dans cette configuration particulière liée à la réalité politique rencontrée, en seconde position, ou encore vient se confondre avec cet impératif d'ordre externe.

\section{Jouissance et maladie circulaire}

L'un des effets psychopathologiques générés par cet impératif à être et demeurer malade serait bien la perpétuation dans le temps de ces affections somatiques. Ce qui implique une sorte de mélancolisation corporelle des douleurs physiques du sujet en raison de la dimension de répétition, d'itérabilité, de durée, voire d'irréversibilité que cette formulation implique. D'ailleurs, et à terme, on observe dans certains cas l'inscription du sujet dans une véritable «position mélancolique ${ }^{25}$ de laquelle s'extraire devient bien difficile.

Dans une perspective d'auto-conservation, le sujet "s'accroche " à sa douleur corporelle qui elle-même s' « agrippe " à lui, rendant circulaire la maladie. D'ailleurs Freud l'avait bien repéré à propos des malades qui souffrent de névrose 
traumatique en disant : " Ils se plaignent de leur maladie, mais I'utilisent de toutes leurs forces et si on veut la leur prendre, ils la défendent comme, d'après le proverbe, la lionne défend son petit. ${ }^{26}$

Chez certains de ces sujets " en mal de refuge " une forme d'enchaînement du corps au symptôme se produit donc, procurant par là même des "poussées " de jouissance continues. Ici, la pulsion ne connait pas d'interdictions et ne limite aucune de ses actions. ${ }^{27}$

Aussi à la jouissance de l'Autre (le souverain), qui incarne une figure de pouvoir et de savoir toute-puissante, le corps du sujet est " attrapé » et répond; une sorte de prise immédiate et directe du discours souverain sur son corps propre s'instaure.

D'une certaine manière, la violence qui provient de l'Autre, en l'occurrence l'État, vient se loger dans son corps. D'ailleurs, la grève de la faim de certains réfugiés déboutés ne vient-elle pas comme une version extrême de la dépossession du corps propre ? Comme une forme d'offrande destinée à ce que le "pays d'accueil» en jouisse?

\section{Le corps dévoilé}

Le corps va "servir " à exprimer quelque chose de l'impensable jadis rencontré à l'autre (les soignants, les assistants sociaux, etc.) mais bien entendu aussi aux interlocuteurs de l'État (Agent de protection de I'OFPRA, Juges assesseurs, etc.) constituant une forme " d'interjection corporelle ${ }^{28}$ qui se donne à voir par le biais $\mathrm{du}$ soma affecté. Le symptôme est aussi une manière de fabriquer de l'Autre, du tiers, quand le sujet névrosé n'arrive plus à exister autrement que dans et par le symptôme.

D'ailleurs, lorsqu'on reçoit ces patients demandeurs d'asile, ils apparaissent souvent égarés par le droit de regard de l'État sur leur corps. Ils se mettent parfois à exhiber ce corps (déjà) juridiquement mis à nu, en nous montrant, à nous soignants, leurs blessures. Cette forme de monstration met mal à l'aise le professionnel acculé à voir ce corps asservi, où la pudeur, telle une peau morte, semble s'être détachée du sujet.

La façon dont le processus de somatisation s'actualise sur le corps interpelle le clinicien qui exerce auprès de réfugiés. Dans cette clinique domine une prééminence des corps qui donne à voir un "raté symbolique" capable de "pousser» les corps à "parler». ${ }^{29} \mathrm{Ce}$ phénomène diffère cependant de la psychosomatique, perspective dans laquelle est trop radicalement séparé le corps de la médecine de la psyché, alors qu'est établi, dans le même temps, une corrélation directe entre ces termes. ${ }^{30}$

Le contexte politique de crise de l'asile favorise donc l'émergence d'un corps malade qui devient le "papier" tant espéré, la carte de séjour du sujet. Objet de convoitise et convoqué par le discours juridique, le corps est instrumentalisé par la preuve, segmenté, et répond par la massivité de sa symptomatologie. À la présence des symptômes psychotraumatiques "classiques" de ces patients se conjuguent donc des affections qui seraient plus directement liées à l'ordre du politique.

Enfin, dans cet entour judiciarisé de " pousse à être malade ", c'est comme si les lésions en voie de cicatrisation devaient se "réouvrir » pour répondre au contexte d'instrumentalisation qui exige presque que le sujet donne à voir des plaies à vif.

Cet aspect n'est pas à concevoir uniquement dans sa dimension métaphorique. Les demandes administratives de preuves seront prises au pied de la lettre par certains demandeurs d'asile qui iront jusqu'à répondre de manière radicale et symptomatique aux autorités du pays d'accueil en s'auto-mutilant par exemple.

Avec ce système " $d$ 'injonction à être marqué ", l'invisible n'a pas lieu d'être, seul le visible est garant et atteste des dommages.

Et lorsque le corps ne témoigne en rien des faits passés - que ce soit en raison des tortures qui ont été effacées par le temps ou l'absence de lésions visibles -, le corps peut devenir un objet persécuteur, lieu de haine et d'acharnement de la jouissance inconsciente du sujet. Quand il 
sent que son corps le "lâche ", le sujet multiplie les investigations médicales pour attester de sa souffrance lancinante dans sa chair, qui demeure inquantifiable. Le travail psychothérapeutique qui s'engage avec le clinicien permettra qu'un nouage entre langage et corps s'opère et qu'ainsi les «pulsions mortifères qui hantent son appareil psyché-soma " estompent les clivages en jeu entre corps et psychisme. ${ }^{31}$

Ces investigations qui portent sur la vie psychique et corporelle des réfugiés n'ont pas pour visée d'attaquer les administrations de l'État. Mais, en revanche, de s'intéresser de près aux effets du politique, et du biopolitique, à partir des achoppements entre les scènes internes - du côté du symptôme- et celles externes - relatives au contexte politique et aux demandes administratives. La porosité entre le psychique et le corporel s'avère éclairante pour penser les territoires humains et politiques, ces topos entrant en interactions constantes, conditionnés par une « impossible étanchéité des frontières ", selon les mots d'Étienne Balibar. ${ }^{32}$

Les avatars de l'articulation de ce système "psyché-soma-preuve " tendent à nous faire penser qu'il y a bien des spécificités à cette clinique de l'asile dans laquelle se télescopent de manière singulière les champs du politique, du psychique et du corporel. Ces formations s'apparentent à une sorte de névrose actuelle où le corps du sujet se trouve en proie à des mutations libidinales.

On voit alors comment se profile pour le demandeur d'asile un corps affecté qui devient un objet double, tel le pharmakon, il incarne le poison et le remède. Il est à la fois l'exil, la douleur mais également la survie, la vie; un refuge.

\section{Bibliographie :}

Agamben, G. (1997). Homo sacer, Le pouvoir souverain et la vie nue (1995). (Raiola, M. trad.) Paris : Le Seuil.

Assoun, P.-L. (2004). Corps et symptôme, Leçons de psychanalyse. Paris : Anthropos/Economica.

Balibar, E. (2002). Droit de cité. Paris : PUF.

Chauvel, S. (2009). Le corps discipliné. Genèses, 2, 75, 2-3. [En ligne] www.cairn.info/revue-geneses-2009-2-page2.htm.

Creac'h, X. (2002). Les évolutions dans l'interprétation du terme réfugié. Revue Hommes et migrations, Les frontières du droit d'asile, 1238, 65.

Crumlish, Niall MSc \& al. (2010). A Systematic Review of Treatments for Post-Traumatic Stress Disorder Among Refugees and Asylum-Seekers. Journal of Nervous \& Mental Disease, 198, 4, 237-251, doi: 10.1097/NMD. Ob013e3181d61258.

Deleuze, G. (2002). Instincts et institutions (1955). L'île déserte et autres textes. Paris : Les Éditions de Minuit.

Douville, O. (2010). Du corporel et de l'incorporel dans la psychose mélancolique. Le corporel, Nouvelles approches en psychosomatique. Paris : Dunod.

Douville, O. \& Galap, J. (1999). Santé mentale des migrants et réfugiés de France. Encyclopédie Médicochirurgicale, Psychiatrie, 37-880-A-10. Paris : Elsevier.

D.S.M. III (1980). American Psychiatric Association. Diagnostic and statistical manual of mental disorders, 3rd ed.. Washington : APA Press

Fassin, D. \& Rechtman, R. (2007). L'empire du traumatisme. Enquête sur la condition de victime. Paris : Flammarion.
Fassin, D. \& Halluin, E. (d') (2009). Critical Evidence: The Politics of Trauma in French Asylum Policies. Ethos, 35, 3, 300-329.

Fazel, M., Doll, H. \& Stein, A. (2009). A school-based mental health intervention for refugee children: an exploratory study. Clinical Child Psychology and Psychiatry, 14(2), 297-309. Oxford University,. [En ligne] http://www.ncbi.nlm.nih.gov/pubmed/19293324.

Fiddian-Qasmiyeh, E. (2010). "Ideal” Refugee Women and Gender Equality Mainstreaming in the Sahrawi Refugee Camps: "Good Practice" for Whom? Refugee Survey Quarterly, 29 (2), 64-84.

Foucault, M. (1975). Surveiller et punir, Naissance de la prison. Paris : TEL Gallimard.

Foucault, M., (2001). La technologie politique des individus (1988). Dits et Ecrits, t. II, 1976-1988. Paris: Gallimard.

Freud, S. (2005). Pour introduire le narcissisme. CEuvres Complètes, XII, (1913-1914). Paris: PUF.

Gushulak, B. D., Weekers, J. \& MacPherson, D.-W. (2010). Migrants and emerging public health issues in a globalized world: threats, risks and challenges, an evidence-based Framework. Emerging Health Threats Journal, 2: e10. doi: 10.3134/ehtj.09.010.

Kirmayer, L.-J. (2003). Failures of imagination: The refugee's narrative. Psychiatry Anthropology \& Medicine, 10, 2, 167-185.

Kirmayer, L.-J. (2011). Defining and delimiting traumarelated dissociation: a view from cultural psychiatry. Journal of trauma \& dissociation, 12(4), 465-468. 
Kirmayer, L.-J. (2002). The refugee's predicament. (Bouville, J.-F. \& Rechtman, R. trad.). L'évolution Psychiatrique, 67, 4, 776-795. Paris : Elsevier.

Le Pors, A. (2011). Le droit d'asile. Paris : PUF.

Lindenmeyer, C. (2012). Qual é o estatuto do corpo na psicanalise ?. Tempo psicanalitico, 44.2. Rio de Janeiro.

Pestre, E. (2007). L'État, le réfugié et son thérapeute. Les conditions de vie psychique des réfugiés. Thèse soutenue en juin 2007 (Paris VII Denis Diderot, dir. Pr. Benslama, F.). Pestre, E. (2010). La vie psychique des réfugiés. Paris: Payot et Rivages.

Pestre, E. \& Benslama, F. (2011). Traduction et traumatisme. Recherches en psychanalyse, 11. [En ligne] www.cairn.info/revue-recherches-en-psychanalyse-2011-

1.htm

Varkey, Prathibha, Kureshi, \& Lesnick, T. (2010). Empowerment of Women and its association with the Health of the Community. Journal of Women's Health \& Gender-Based Medicine, 19 (1), 71-76.

\section{Notes :}

${ }^{1}$ Il s'agit de l'OFPRA (Office Français de Protection des Réfugiés et Apatrides) et de la CNDA (Cour Nationale du Droit d'Asile), ex-CRR (Commission des Recours des Réfugiés).

${ }^{2}$ Ces preuves corporelles ne sont pourtant pas exigées par la Convention de Genève ni par les textes qui la complètent : aucun ne mentionne la nécessité de montrer des " preuves de tortures" pour accéder à la qualité de réfugié. Le protocole d'Istanbul en témoigne : "Lorsqu'il existe des preuves physiques de la torture, celles-ci apportent une confirmation importante desdites déclarations. Toutefois, l'absence de telles preuves ne devrait pas être invoquée pour nier la torture, car de nombreuses formes de sévices ne laissent pas de traces et encore moins de cicatrices permanentes. " Protocole d'Istanbul, Haut-commissariat des Nations Unies aux droits de I'homme, 2001, Manuel pour enquêter efficacement sur la torture et autres peines ou traitements cruels, inhumains et dégradants. Série sur la formation professionnelle $n^{\circ} 8$. p. 34-47.

${ }^{3}$ Les taux d'obtention de l'asile s'avèrent extrêmement bas: moins d'un quart des demandeurs a obtenu la qualité de réfugié en 2010 (52 762 demandes contre seulement 10340 acceptées). Ces chiffres donnés par I'OFPRA sont notamment repris par Le Pors, A. (2011). Le droit d'asile. Paris : Que sais-je, PUF (4è édition).

${ }^{4}$ Depuis une décennie, les propos des ministres de I'Intérieur vont dans ce sens. D. de Villepin évoquait en 2003 le dispositif "engorgé » de l'asile, "source de détournements de procédure ». C. Guéant réutilisait en 2011 l'expression de "détournements de procédures ", celles-là même qui, selon lui, "saturent la machine administrative, au détriment des personnes qui peuvent légitimement revendiquer le statut de réfugié politique en France ", cité par Le Figaro, quotidien du 25/11/2012.

${ }^{5}$ Pourtant, cette terminologie a été abandonnée peu après la Seconde Guerre Mondiale - Consulter Creac'h X. Les évolutions dans l'interprétation du terme réfugié. Revue Hommes et migrations, Les frontières du droit d'asile, n¹238, juillet-août 2002, p. 65.

${ }^{6}$ Gushulak, B. D., Weekers, J. \& MacPherson, D.-W. (2010). Migrants and emerging public health issues in a globalized world: threats, risks and challenges, an evidence-based Framework. Emerging Health Threats Journal, 2: e10. doi: 10.3134/ehtj.09.010.

${ }^{7}$ Les études médicales anglo-saxonnes qui portent sur la santé des réfugiés sont généralement d'ordre descriptif, comparatif et spécifique. On répertorie de nombreux articles portant sur le genre, notamment sur l'étude des violences faites sur les femmes réfugiées ou sur la santé mentale des enfants. Cons. Notamment: Fazel, M., Doll, H. \& Stein, A. (2009). A school-based mental health intervention for refugee children: an exploratory study. Oxford University, Apr;14(2), 297-309.

${ }^{8}$ Le PTSD a fait son apparition dans le D.S.M. III, (1980). American Psychiatric Association. Diagnostic and statistical manual of mental disorders, 3rd ed., Washington: APA Press, p. 256. En 1996 (DSM IV) pour que soit formulé ce diagnostic quatre critères doivent être repérés (la confrontation à l'événement traumatique, symptômes d'intrusion, symptômes d'évitementémoussement, symptômes neurovégétatifs).

${ }^{9}$ Crumlish, Niall MSc, 1 al. (April 2010). A Systematic Review of Treatments for Post-Traumatic Stress Disorder Among Refugees and Asylum-Seekers. Journal of Nervous \& Mental Disease, Volume 198, Issue 4, p. 237-251, doi: 10.1097/NMD.0b013e3181d61258.

${ }^{10}$ Fassin, D. \& Rechtman, R. (2007). L'empire du traumatisme. Enquête sur la condition de victime. Paris : Flammarion.

${ }^{11}$ Varkey, Prathibha, Kureshi, \& Lesnick, T. (2010). Empowerment of Women and its association with the Health of the Community. Journal of Women's Health \& Gender-Based Medicine, 19 (1), 71-76.

${ }^{12}$ Fiddian-Qasmiyeh, E. (2010). "Ideal" Refugee Women and Gender Equality Mainstreaming in the Sahrawi Refugee Camps: “Good Practice” for Whom?”. Refugee Survey Quarterly, 29 (2), p. 64-84.

${ }^{13}$ Kirmayer, L.-J. (2011). Defining and delimiting traumarelated dissociation: a view from cultural psychiatry. Journal of trauma \& dissociation, 12(4), 465-468; (2003) Failures of imagination: The refugee's narrative. Psychiatry Anthropology \& Medicine, Volume 10, Issue 2, p. $167-185$; (2002) The refugee's predicament (Bouville, J.-F. \& Rechtman, R. trad.). L'évolution Psychiatrique, 67, $\mathrm{n}^{\circ} 4$, p.776-795. Elsevier. 
${ }^{14}$ Pestre, E. (2010). La vie psychique des réfugiés. Paris: Payot et Rivages.

${ }^{15}$ Douville, O. \& Galap, J. (1999). Santé mentale des migrants et réfugiés de France. Encyclopédie Médicochirurgicale, Psychiatrie, 37-880-A-10. Paris : Elsevier.

${ }^{16}$ Cette recherche a fait l'objet d'une thèse: Pestre, $E$. (2007). L'État, le réfugié et son thérapeute. Les conditions de vie psychique des réfugiés, soutenue en juin 2007 (Paris VII, direction Pr. Benslama F.).

${ }^{17}$ Agamben, G. dans Homo sacer, écrit à propos de l'approche foucaldienne du bio-pouvoir: "Le pouvoir pénètre dans le corps même des sujets et dans leurs formes de vie ", Op. cit., p. 13.

${ }^{18}$ Foucault, M. (2001). La technologie politique des individus (1988). Dits et Ecrits, t. II, 1976-1988. Paris: Gallimard, Coll. Quarto, p. 1632-1647, p. 1643 ; Surveiller et punir (1975). Naissance de la prison. Paris: TEL Gallimard.

${ }^{19}$ Comité Médical d'Aide aux Réfugiés. Ce centre, situé à l'hôpital du Kremlin-Bicêtre est aussi un observatoire de la santé des réfugiés en France.

${ }^{20}$ Consulter à ce propos: Fassin, D. \& Halluin, E. (d') (2009). Critical Evidence: The Politics of Trauma in French Asylum Policies. Ethos, 35, 3, 300-329, Favell, Adrian.

${ }^{21}$ Chauvel, S. (2009). Le corps discipliné. Genèses, 2, n 75 , p. 2-3. [En ligne] www.cairn.info/revue-geneses-2009-2page-2.htm.

${ }^{22}$ Article L.313-11 du Code de l'entrée et du séjour des étrangers et du droit d'asile. Ce droit au séjour (APS) s'arrête lorsque le patient est " guéri », ce qui notamment s'avère problématique chez les patients qui souffrent de pathologies chroniques.
${ }^{23}$ Freud, S.. Pour introduire le narcissisme. CEuvres Complètes, Vol. XII, (1913-1914), p. 213-247.

${ }^{24}$ Assoun, P.-L. (2004). Corps et symptôme, Leçons de psychanalyse, $2^{\text {ème }}$ édition. Paris : Anthropos/Economica, p. 58. ${ }^{25}$ Douville, O. (2010). Du corporel et de l'incorporel dans la psychose mélancolique. Le corporel, Nouvelles approches en psychosomatique. Paris: Dunod, Coll. Inconscient et culture.

${ }^{26}$ Freud, S. (1926). La question de l'analyse profane, chapitre V, CEuvres complètes, Vol. XVIII. Paris : PUF, p. 46. ${ }^{27}$ Je reprends ici en substance les propos de Deleuze $G$. dans son rapprochement entre "Instincts et institutions", si on envisage l'instinct en terme de pulsion, Deleuze, G. (2002). Instincts et institutions (1955). L'île déserte et autres textes. Paris : Les Éditions de Minuit.

${ }^{28}$ Assoun, P.-L. (1999). Le préjudice et l'ldéal, Pour une clinique sociale du trauma. Paris: Antropos/Economica, p. 293.

${ }^{29}$ Assoun, P.-L. (2004). Corps et symptôme, Leçons de psychanalyse, Ibid, p. 27.

${ }^{30} \mathrm{Je}$ renvoie, pour plus de développements épistémologiques sur la perspective psychosomatique et le statut du corps dans la psychanalyse, au texte de Lindenmeyer, C. (2012). Qual é o estatuto do corpo na psicanalise?. Tempo psicanalitico, 44.2. Rio de Janeiro. (PsycolNFO)

${ }^{31}$ Pestre, E. \& Benslama, F. (2011). Traduction et traumatisme. Recherches en psychanalyse, 11 [En ligne] www.cairn.info/revue-recherches-en-psychanalyse-20111.htm

${ }^{32}$ Balibar, E. (2002). Droit de cité. Paris: PUF, Coll. Quadrige, p. 87.

\section{L'auteur :}

\section{Élise Pestre}

Psychologue clinicienne et psychanalyste.

Docteur en psychopathologie, Maître de

conférence en psychopathologie à l'Université

Paris VII Diderot, "Politique de la santé et des

minorités ", Centre de Recherche en

Psychanalyse, Médecine et Société, Lab (EA

3522).

Université Paris VII Diderot

Campus Paris Rive Gauche

Bâtiment Olympe de Gouges

11, rue Jean Antoine de Baïf

75013 Paris

France

\section{Référence électronique}

Élise Pestre, "L'instrumentalisation par la preuve du corps du réfugié ", Recherches en Psychanalyse [En ligne], 14|2012, mis en ligne le 28 décembre 2012.

Texte intégral

Droits d'auteur

Tous droits réservés 